\section{La apendicitis y su historia}

\author{
PABLO YOUNG
}

\section{Appendicitis and its history}

Appendicitis is a common cause of acute abdominal pain. It is caused by the obstruction of the cecal lumen and can evolve to perforation and peritonitis. This article reviews the initial description and history of appendicitis and describes the signs and symptoms used for diagnosis. In today's medicine, where technology plays an important role, the value of anamnesis and physical examination should be stressed as useful tools.

(Rev Med Chile 2014; 142: 667-672)

Key words: Appendicitis; History of medicine; Signs and symptoms, digestive.

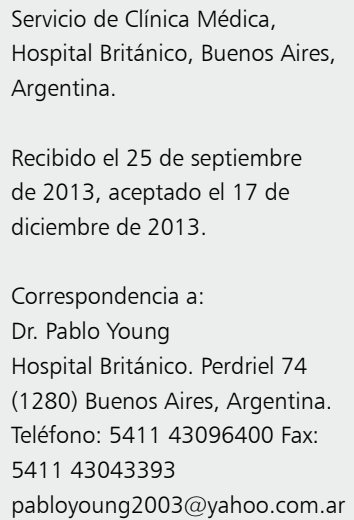

L a apendicitis (AP) constituye un proceso inflamatorio agudo iniciado por la obstrucción de la luz apendicular cecal y que, librado a su evolución espontánea, puede originar la perforación con la consiguiente peritonitis. Se estima que $5 \%$ de la población la padecerá en algún momento de su vida. En 1886 fue reconocida como entidad clínica y anatomopatológica por Reginald Heber Fitz (1843-1913)1 (Figura 1).

El apéndice fue descripto por Berengario DaCarpi (1460-1530) (Figura 2) en el año 1521, aunque se observó claramente en las descripciones de anatomía de Leonardo Da Vinci (1452-1519), hechas en 1492, pero fueron publicadas en el siglo 18 (Figuras 3 y 4). También se encuentran en ilustraciones de Andreas Vesalius (1514-1564) en su libro "De Humani Corporis Fabrica" publicado en 1543 (Figura 5) ${ }^{2}$.

La inflamación aguda del apéndice vermiforme es probablemente tan vieja como el hombre. En momias egipcias de la era Bizantina se observaron adhesiones en el cuadrante inferior derecho, sugestivas de AP antiguas ${ }^{2}$. Si bien se ha adjudicado su descripción a Jean Fernel y Von Hilden, fue Lorenz Heister (1683-1758) discípulo de Hermann Boerhaave (1668-1738) quien describió una AP perforada con absceso en 1711. La descripción de un fecalito dentro de una AP perforada fue publicada por James Parkinson (1755-1824) en el año 1812. En 1824, Louyer-Villermay (1707-1770) describió la AP gangrenosa en un artículo presen- tado ante la Real Academia de Medicina de Paris y así estimuló el interés de Francois Melier, médico parisino, quien en 1827 propuso la remoción del apéndice como tratamiento de esta entidad. Se dice que el artículo de Melier fue ignorado durante mucho tiempo por la confrontación que tenía con el gran cirujano parisino Barón Guillaume Dupuytren (1777-1835) $)^{2,3}$.

En el primer volumen del libro "Elementos de medicina práctica" publicado en 1839 , los doctores Richard Bright (1789-1858) y Thomas Addison (1793-1860) del Guy's Hospital, describieron la sintomatología de la AP y establecieron que la AP provoca la mayoría de los procesos inflamatorios de la fosa ilíaca derecha ${ }^{3}$.

En junio de 1886 se realizó el congreso de la Asociación Americana de Médicos en Washington, DC. Muchos líderes de la medicina americana estuvieron presentes, como Sternberg, Prudden, Osler y otros. El 18 de junio el Dr. Reginald H. Fitz presentó su conferencia titulada "Perforating inflammation of the vermiform appendix; with special reference to its early diagnosis and treatment". En este escrito, Fitz enfatizó que el origen de la mayoría de los procesos inflamatorios de la fosa ilíaca derecha están en el apéndice. Describe con claridad el cuadro clínico y lo más sorprendente es que propone la cirugía temprana como tratamiento, siendo además el primero que utilizó el término apendicitis ${ }^{1,2}$.

Reginald H Fitz perteneció a la novena ge- 


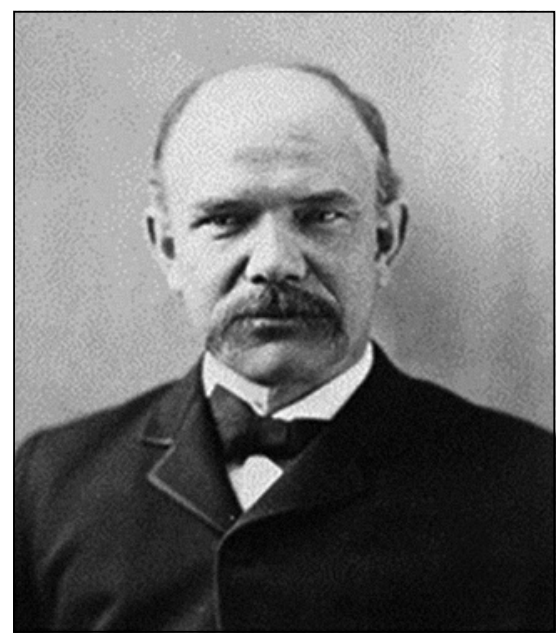

Figura 1. Reginald H. Fitz. En: http://oasis. lib.harvard.edu/oasis/deliver/ med00049/; consultado el 31/5/14.

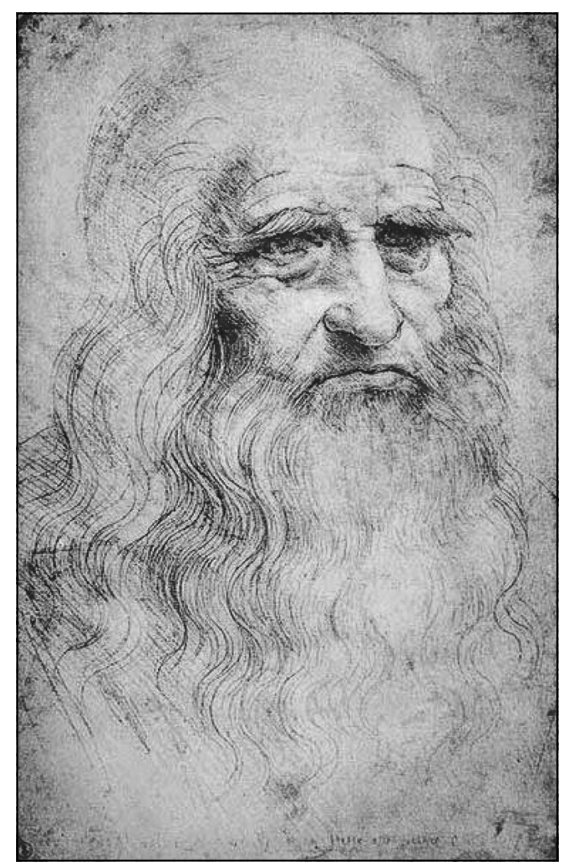

Figura 3. Leonardo Da Vinci. En: http://boingboing.net/2011/09/14/the-culinary-notebooksof-leonardo-fat-boy-da-vinci.html; consultado 12/9/2013.

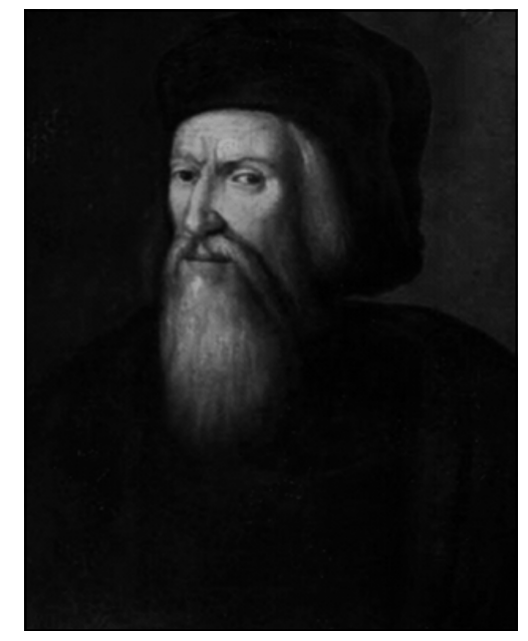

Figura 2. Berengario DaCarpi. En: http://himetop.wikidot. com/jacopo-berengario-dacarpi-s-portrait/; consultado el 30/5/14.

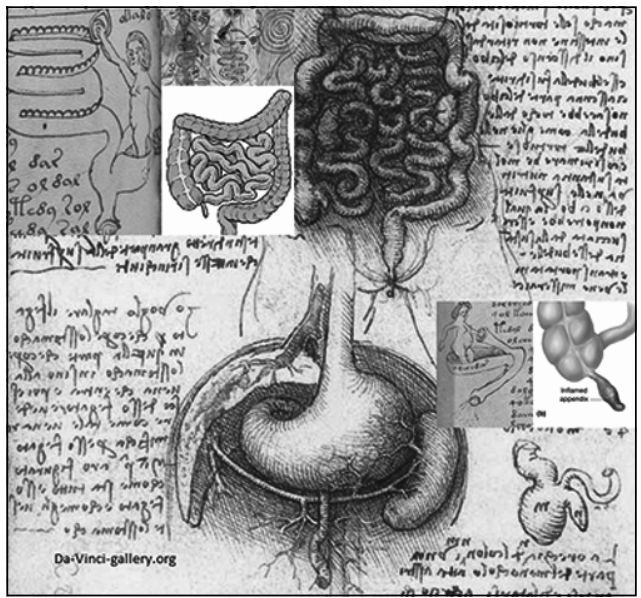

Figura 4. Leonardo Da Vinci, descripción del apéndice. En: http:// ellievelinska.blogspot.com/2013/06/ the-voynich-manuscript-da-vincis. html; consultado 31/5/2014.

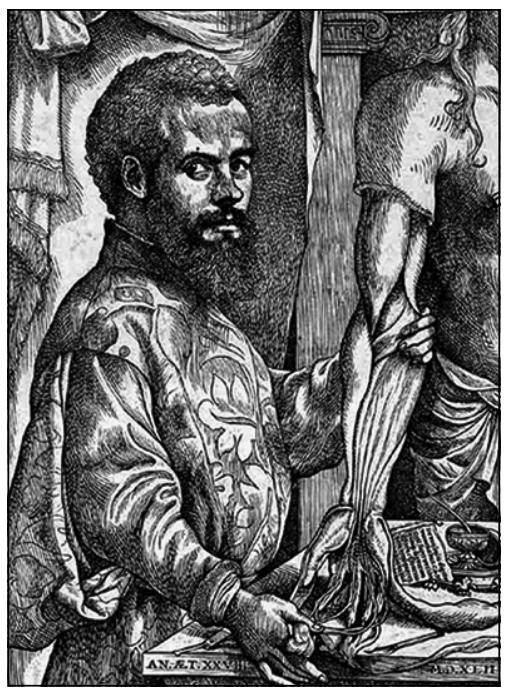

Figura 5. Andrés Vesalio (retrato procedente de su obra Fabrica). En: http://es.wikipedia. org/wiki/Andr\%C3\%A9s_Vesalio; consultado 31/5/2014. 
neración de Fitz en América, nació en Boston, estudió en el Harvard College y posteriormente en Harvard Medical School y realizó un año de prácticas en el Boston City Hospital. Continuó su formación en diferentes hospitales de Viena, Berlín, Paris, Londres y Glasgow, durante dos años. En Viena aprendió patología celular con Rudolf Virchow (1821-1902). En 1870 regresó a Boston e ingresó como instructor de anatomía patológica de la Universidad de Harvard ${ }^{3}$.

Fitz sostuvo su proposición con las autopsias de 257 casos de perforaciones del apéndice que comparó con 209 casos de tiflitis y peritiflitis. Sobre la etiología de la apendicitis sus consideraciones son casi las mismas que aún se discuten.

La muerte en la apendicitis es por shock después de la peritonitis y el proceso puede extenderse al mesenterio. En 11 casos de los 257 Fitz encontró pileflebitis. Fitz no dudó de que no todas las apendicitis perforadas sean fatales. De las tiflitis y peritiflitis, 58 terminaron en resolución (32\%), 33 por evacuación espontánea $(18 \%)$ y 89 por operación $(50 \%)$.

Fitz terminó su conferencia con estas afirmaciones: "La vital importancia del diagnóstico temprano de la apendicitis perforada es obvia. El diagnóstico, en la mayoría de los casos, es comparativamente fácil. El eventual tratamiento por laparotomía es generalmente indispensable. Síntomas urgentes demandan la inmediata exposición del apéndice perforado, después de la recuperación del shock, $y$ su tratamiento de acuerdo con los principios quirúrgicos. Si la espera se justifica, el absceso resultante, por regla intraperitoneal, debería ser incindido tan pronto como sea evidente. Esto es usualmente en el tercer día después de la aparición de los primeros sintomas característicos de la enfermedad". Los términos "tiflitis" y "peritiflitis" se han desechado gradualmente, aunque el término tiflitis se sigue utilizando sólo para denominar la inflamación del ciego en el contexto de la colitis neutropénica, entre otros.

Al tiempo de la presentación de su artículo era profesor de anatomía patológica de la Universidad de Harvard y médico del Massachusetts General Hospital. Continuó activo en su trabajo hospitalario hasta antes de su muerte, a la edad de 70 años, luego de una cirugía de úlcera gástrica.

Sir Frederick Treves (1893-1923), cirujano, escritor e historiador inglés, contribuyó a la difusión de las ideas de Fitz. Su influyente conferencia
"Relapsing tyflitis treated by operation", cuyo texto recibió la Royal Medical and Chirurgical Society en septiembre de 1887, se leyó en febrero de 1888 y se publicó en el mismo año. Treves fue quien, en 1902, operó de apendicitis a Eduardo VII tres días antes de la programada coronación, desde luego pospuesta, y quien cuidó a Joseph Merrick, el "Hombre Elefante"4.

La primera vez que se removió el apéndice quirúrgicamente fue en 1735 por el Dr. Claudius Amyand (1681-1740) fundador del St. George's Hospital y cirujano del Westminster Hospital de Londres ${ }^{2,3}$. Amyand nació en París, en una familia de hugonotes de la Santoigne que huyó a Inglaterra. Aymand sirvió como cirujano militar en Flandes, en 1716 fue incorporado a la Royal Society. Fue cirujano en el St. George's Hospital y Sergeant-Surgeon (Surgeon-in-Ordinary) (17151740) del rey George I (1714-1727) y luego de George II (1727-1760) ${ }^{3}$.

Amyand relató que el 8 de octubre de 1735 ingresó al St. George's Hospital Hanvil Anderson, un chico de 11 años, que desde la infancia tenía una hernia escrotal, complicada con una fístula entre el escroto y el muslo que drenaba gran cantidad de materia fecal y que para curarla era necesario curar la hernia. Lo operó el siguiente 6 de diciembre. El tumor estaba compuesto en su mayor parte por epiplón, tenía el tamaño de una manzana y en él se encontraba el apéndice cecal perforado en la punta por un alfiler incrustado en una concreción calcárea; por la perforación salía materia fecal cada vez que se movía el alfiler adherido al epiplón. Alrededor, un absceso incluía el conducto deferente y el testículo contenidos en el saco herniario. Amyand no sabía qué hacer con el intestino perforado que reconoció, al final, como el apéndice cecal "contraído, carnoso y duplicado". Tras una complicada disección, extendida a la cavidad abdominal, resecó el epiplón, separó los vasos, el deferente y el testículo y, de acuerdo a la opinión de los asustados concurrentes, decidió amputar el anormal apéndice tras una ligadura en la base. Retornó el intestino a la cavidad abdominal, dejó el resto de los elementos en el saco escrotal y resecó la fístula. La incisión se mantuvo abierta con una compresa. La operación duró casi media hora y fue "tan dolorosa para el paciente, como laboriosa para mí”. Al mes el chico fue dado de alta. La fístula curó, la hernia recidivó a los seis meses $^{3,5}$. Las apendicitis dentro de las hernias no 
son tan infrecuentes, ocurriendo en hernias inguinales (hernia de Amyand) y femorales (hernia de Garengeot).

La teoría de Dieulafoy se refiere a que la AP se debe a la oclusión de la cavidad apendicular. Además de describir la teoría, Georges Paul Dieulafoy (1839-1919) describió la enfermedad de Dieulafoy o lesión de Dieulafoy, que es una anomalía vascular localizada generalmente en el estómago y consiste en la presencia de una arteria de gran calibre en la submucosa, y ocasionalmente en la mucosa, de evolución muy aguda y brusca con hematemesis abundantes que sobreviven en plena salud sin pródromos gástricos. También se llama así a la forma tóxica de la apendicitis. Se conoce como "drama pancreático de Dieulafoy", referido también como un disparo en la noche serena a la aparición brusca de síntomas, muy alarmantes (dolor epigástrico, vómitos repetidos, meteorismo abdominal, estado de shock) al comienzo de una pancreatitis aguda hemorrágica.

John Benjamin Murphy (1857-1916) en 1889, realizó la primera cirugía temprana para prevenir las complicaciones de la apendicitis ${ }^{2}$.

El cuadro clínico inicial de la apendicitis aguda se presenta por lo general, ordenado y cronológico, según se describe en la triada sintomática de Murphy. Esta no es patognomónica de la apendicitis ya que se puede observar en la pancreatitis y la úlcera perforada. Está constituida por dolor epigástrico o peri umbilical, que es un síntoma capital, casi siempre llamativo y alarmante, seguido de anorexia, náuseas o vómitos; el dolor se irradia y se localiza en la fosa ilíaca derecha, a esto se le puede agregar fiebre y leucocitosis, si esto último ocurre, se la llama péntada de Murphy. Se presenta aproximadamente en $60 \%$ de los pacientes. El dolor provocado al descomprimir bruscamente la fosa iliaca derecha se denomina signo de Blumberg en honor a su descubridor Jacob Moritz Blumberg (1873-1955), y si es generalizado se llama signo de Noël Guéneau de Mussy (1813-1885).

El dolor se presenta de manera aguda, suele ser continuo, aunque a veces puede presentarse como cólico; puede comenzar en el epigastrio, se acompaña siempre de dolor a la presión, profunda o superficial, en toda la región de la fosa ilíaca derecha, pero especialmente en el punto de MacBurney lo que tiene una sensibilidad de 63\% y especificidad $69 \%$. En este punto, la piel puede estar hiperestésica, haciendo insoportable el solo contacto con los dedos del explorador. A esta zona de hipersensibilidad en fosa ilíaca derecha, debida a distensión del apéndice, se la ha llamado triángulo apendicular de Sherren. Estas características en conjunto se las ha llamado tríada de Dieulafoy, compuesta por dolor a la palpación, hiperestesia cutánea y defensa en fosa ilíaca derecha. En cuanto al dolor, es importante recordar a los antiguos cirujanos que decían "si aparece primero dolor y luego el vómito, el cuadro es quirúrgico"11.

Charles Heber McBurney (1845-1913), profesor de cirugía en el College of Physicians and Surgeons (Columbia University) de New York describió el sitio preciso del dolor en 1889 y la incisión adecuada para exponer y extirpar el apéndice inflamado en $1894^{2,3}$. Casi 90 años después, en 1981, el Dr. Kurt Semm (1927-2003) efectuó la primera apendicectomía laparoscópica, de esta manera se inició la era laparoscopica de la cirugia ${ }^{12}$.

Los vómitos siguen a la iniciación del dolor de 1-4 h, nunca lo preceden, este fenómeno tiene una sensibilidad de $100 \%$ y una especificidad de $64 \%$. Este síntoma puede faltar o tener su equivalente en el estado nauseoso que con frecuencia se observa con variable intensidad, desde sólo un vómito hasta la repetición alarmante de las formas graves ${ }^{13}$.

Los antiguos clínicos describían en la evolución de algunos pacientes, un período en el cual los síntomas, en especial el dolor, desaparecían haciendo poner en duda el diagnóstico. A esta situación clínica la reconocían como la "calma traidora" o el "Paraíso de los tontos". La explicación fisiopatológica de este fenómeno podían ser dos, la primera es que el dolor por la distensión inflamatoria o las contracciones causadas por la obstrucción del apéndice cede cuando éste se perfora y vacía su contenido a la cavidad abdominal. Esto provocaría un período variable de bienestar que obviamente iba seguido de un recrudecimiento del cuadro doloroso. La otra explicación es que el proceso inflamatorio séptico progrese a la isquemia y a la necrosis de la pared apendicular, comprometiendo las terminaciones nerviosas de los plexos intramurales responsables de recoger la sensibilidad visceral y de este modo se interrumpe la señal. En ambas condiciones es requisito que el apéndice inflamado no esté en contacto con el peritoneo parietal que transmite información dolorosa por la vía espinal. Por lo demás, en las dos situaciones se asiste a una progresión y agravamiento del cuadro y por eso los autores eran 
particularmente cautos en la interpretación de esta aparente mejoría. La desaparición del dolor y el Blumberg, si no ocurre junto "con la mejoría de todos los otros síntomas", indica más bien que el apéndice se ha gangrenado o que la inflamación ha progresado a los tejidos vecinos y por lo mismo la cirugía no debe posponerse ${ }^{14}$.

En etapas tempranas de la enfermedad, los síntomas y signos son poco llamativos y dependen de la posición del apéndice; por ejemplo, un apéndice ubicado en la fosa ilíaca derecha, descendente interno, anterior, dará lugar incipiente a defensa muscular y dolor con la compresión en dicha fosa. $\mathrm{Si}$, por el contrario, el apéndice se encuentra en la pelvis, el dolor en dicha fosa ilíaca será mínimo y debemos buscarlo por medio del tacto rectal o vaginal. El tacto rectal o vaginal no deben obviarse, ya que la palpación en la pared lateral derecha del recto o vagina puede ser dolorosa. Además no solo permiten confirmar el diagnóstico sino también establecer la existencia o no de otras afecciones que puedan simular una apendicitis aguda. En este punto es propicio recordar la frase acuñada por Hamilton Bayley, quien decía "que por no meter el dedo se mete la pata" ${ }^{10}$. Además, el tacto rectal es de utilidad en los pacientes con contractura de la pared abdominal, pues permite localizar el sitio de lesión. Para ello se utiliza la maniobra de Yódice Sammartino (palpación anoparietoabdominal). En los apéndices pelvianos, Handley ha descripto un cuadro que denominó íleo doble, que es la obstrucción del intestino delgado y el colon sigmoides al mismo tiempo ${ }^{6-15}$.

Si está en contacto con la vejiga provocará polaquiuria o disuria. Si lo está con el recto, el paciente referirá pujos y tenesmo. Un apéndice retrocecal extraperitoneal, provocará escaso dolor en la fosa ilíaca derecha, pero se manifestará por dolor a la palpación en la zona lumbar ${ }^{15}$.

Si está ubicado en la fosa ilíaca derecha, por detrás de las asas intestinales, las manifestaciones locales (defensa y contractura) son pobres y el compromiso del tránsito intestinal hará que la afectación apendicular se exprese por un síndrome oclusivo del intestino delgado ${ }^{6-10}$.

Cuando el proceso adquiere mayores proporciones, hasta alcanzar el peritoneo parietal anterior, se produce la contractura de los músculos vecinos, corrientemente designada "defensa muscular”. Es válido recordar que la contractura de los músculos anchos cuando es producido por un proceso inflamatorio como en la AP, cede escalonadamente cuando la presión de la mano que palpa es sostenida, "defensa por etapas de Oudart", contrariamente al vientre en tabla que es irreductible y expresa un profundo y sorpresivo agravio al peritoneo, como proceso en la irritación química de la serosa en la úlcera perforada de estómago o duodeno $^{11,13}$. Se han descripto más de 60 epónimos relacionados al examen físico en la AP.

A través de la historia, los médicos se han dedicado a encontrar pistas que conduzcan a un diagnóstico correcto en el caso de una patología tan común como la apendicitis.

En la medicina de hoy, donde la tecnología juega un rol importante, como dice el Dr. Agrest ${ }^{16}$, "hemos llegado a escuchar poco, explicar menos, pedir muchos exámenes auxiliares y decidir sobre la base de lo que los exámenes nos informan", perdiendo así el valor relevante de la anamnesis y el examen físico. La semiología, la anamnesis y el contacto físico constituyen las bases no sólo del diagnóstico sino también de la relación médico paciente. El abandono de estos recursos hará perder humanidad en la actividad médica, el placer de resolver un diagnóstico con una pregunta oportuna, con una auscultación que nadie ha hecho, con una palpación que descubre dolor donde las ecografías, tomografías y resonancia no habían demostrado anormalidad. Quizás no estamos asistiendo al ocaso de la semiología, sino al renacer de una nueva semiología, que pone el esfuerzo en el arte de descubrir lo que los recursos auxiliares no consiguen hacer o lo hacen con mayor agresividad, con mayor riesgo y siempre con mayor costo y diluyendo responsabilidades ${ }^{17}$.

\section{Referencias}

1. Fitz RH. Perforating inflammation of the vermiform appendix; with special reference to its early diagnosis and treatment. Am J Med Sci 1886; 92: 321-46.

2. Williams GR. Presidential address: a history of apendicitis. With anecdotes illustrating its importance. Ann Surg 1983; 197: 495-506.

3. Barcat JA. Sobre la apendicitis aguda: Amyand, Fitz, y unos pocos más. Medicina (B Aires) 2010; 70: 576-8.

4. Treves F. Relapsing typhlitis treated by operation. Med Chir Trans 1888; 71: 165-72.

5. Amyand C. Of an Inguinal Rupture, with a Pin in the Appendix Coeci, Incrusted with Stone; And Some Ob- 
servations on wounds in the guts. Philos Trans R Soc Lond 1736; 39: 329-42.

6. Ocampo C. Apéndice cecal. En: Ferraina P, Oria A. Cirugía de Michans. El Ateneo editores, Buenos Aires: 1997, p 806-15.

7. Pieper R, Kager L. The incidente of acute appendicitis and appendectomy: an epidemiological study of 971 cases. Acta Chir Scand 1982; 148: 45-51.

8. Humes DJ, Simpson J. Acute appendicitis (Clinical review). BMJ 2006; 333: 530-4.

9. Birnbaum BA, Wilson SR. Appendicitis at the Millennium. Radiology 2000; 215: 337-48.

10. Waisman H, Gutiérrez D. Apendicitis aguda. En: Waisman H, Gutiérrez D. Abdomen Agudo. EDIMED editores, Buenos Aires: 1984, p 42-8.

11. Cope Z. Apendicitis. En: Cope S. Diagnóstico precoz del abdomen agudo. Marin editores, $12^{\text {th }} \mathrm{ed}$. Barcelona: 1947, p 65-82.

12. Bhattacharya K. Kurt Semm: A laparoscopic crusader. J Minim Access Surg 2007; 3: 35-6.

13. Paulson EK, Kalady MF, Papps TN. Suspected Appendicitis. N Engl J Med 2003; 348: 236-42.

14. Korn O. Apendicitis aguda sin dolor o "El paraíso de los tontos". Caso clínico. Rev Med Chile 2008; 136: 1559-63.

15. Marañón G. Abdomen Agudo. En: Manual de Diagnóstico Etiológico. Espasa-Calpe, editores, $5^{\mathrm{a}}$ ed. Madrid: 1950, P 239-53.

16. Agrest A. Pasado, presente y futuro en la enseñanza de la medicina clínica. En: Avances en medicina '94. Cámera MI, Romani A, Madoery C, Farías J, editores, Buenos Aires: Sociedad Argentina de Medicina; 1994, p 295306.

17. Agrest A. El ocaso de la semiología. Medicina (B Aires) 2008; 68: 175. 